\title{
A CRECHE COMO CONTEXTO SOCIO-AFETIVO DE DESENVOLVIMENTO: OS PADROES INTERACIONAIS E O AJUSTAMENTO DAS CRIANÇAS
}

\author{
DAY-CARE CENTRES AS A SOCIAL AND AFFECTIVE \\ DEVELOPMENTAL CONTEXT: INTERACTIONAL PATIERNS \\ AND THE ADJUSTMENT OF PRE-SCHOOL CHILDREN
}

Vera Silvia Raad Bussab ${ }^{1}$

Maria Paula Costacurta Maluf ${ }^{2}$

BUSSAB, V.S.R.; MALUF, M.P.C. A Creche como Contexto Sócio-Afetivo de Desenvolvimento: Os Padrões Interacionais e o Ajustamento das Crianças. Rev. Bras. Cresc. Desenv. além., São Paulo, 8 (1/2), 1998.

Resumo: O estudo do comportamento de crianças em creches tornou-se de fundamental importancia na vida contemporânea, em virtude da substancial adesão a esse modo de criação e da compreensão da importância do contexto sócio-afetivo cotidiano para o desenvolvimento do individuo. A presente pesquisa visou estudar os tipos de interações sociais de crianças de 4 anos, em uma creche pública estadual, com o objetivo de estabelecer relações entre os vários comportamentos das crianças e identificar possíveis padrões de ajustamento social e afetivo à creche. Foram feitas gravações em video, pelo método sujeito focal, de 22 crianças em recreação livre, em 6 sessões de 10 minutos para cada sujeito, distribuídas ao longo de 2 meses. Os dados da totalização das frequências de cada categoria observada foram analisados estatisticamente através de testes de correlação de Pearson e de testes t de comparação de médias. Estas análises revelaram a existência de padrões interacionais definidos, especialmente em função do nivel de ir^lteruçõés positivas. Crianças que se envolveram em poucas interações positivas tenderam a apresentar uma distribuição tipica dos demais comportamentos, oposta à verificada no caso das crianças que interagiram muito. Níveis baixos de interações positivas apareceram correlacionados com: 1- níveis altos de observar os demais, o que enfraquece uma suposição de desinteresse social como explicação para a falta de intonações positivas; 2- níveis altos de apatia, sugestivos de dificuldade no ajustamento à situação; 3- níveis baixos de alguns padrões agressivos. No presente estudo, estes efeitos não foram modulados por tempo de creche, talvez pela ausência de tempos menores do que 2 anos, e nem por sexo, possivelmente pela complexidade de interação desta variável com as demais.

Palavras-chave: creche; contexto de desenvolvimento; interação; ajustamento infantil.

1 Professora-Doutora do instituto de Psicologia da USP. End.: R. Poconé 181 - São Paulo - SP - CEP 01254-040. Bolsa de produtividade de pesquisa do CNPq.

2 Aluna de Graduação do instituto de Psicologia daUSP. End.: R. Pde. João Manuel 422 - São Paulo - SP - CEP 01411-000. Instituto de Psicologia/USP. Bolsista de iniciação Científica/PIBIC do CNPq. 


\section{INTRODUÇÃO}

No modo de vida urbano contemporâneo, as famílias têm optado, cada vez mais, por colocar seus filhos pequenos sob os cuidados de creches ou escolinhas, durante um ou dois períodos diurnos. Em virtude das demonstrações da importância do contexto sócio-afetivo cotidiano da criança para o seu desenvolvimento, tem ficado evidente a relevância dos estudos que visam compreender a dinâmica das relações das crianças nessa situação e que buscam promover condições adequadas para o desenvolvimento infantil nessas instituições.

Pesquisas longitudinais baseadas na teoria de apego de BOWLBY (1984) têm demonstrado que o tipo de vínculo afetivo que uma criança estabelece inicialmente com a mãe influencia de modo geral seu desenvolvimento subsequente e suas relações com outros adultos e crianças. Assim, crianças classificadas como inseguras quando bebês, tendem a ser mais agressivas na creche aos 4 anos (BOOTH et al., 1991); este efeito aparece de modo mais proeminente nos meninos, que podem ser menos apreciados pelos colegas e indicados como problemáticos pelos educadores, tanto na pré-escola, (COHN, 1990), quanto na escola primária (RENKEN et al., 1989). A amizade entre díades de crianças também repete o estilo de vinculação, pois duplas em que ambas as crianças têm apego seguro são mais positivas e coordenadas (KERNS et al., 1995). Pode-se dizer ainda que o desempenho geral também é afetado. Crianças previamente classificadas como seguramente apegadas tendem a se mostrar mais entusiastas, persistentes, cooperativas e eficientes nas tarefas do que as demais (MATAS et al., 1978).

È interessante notar que o desenvolvimento de vínculos afetivos na creche também parece ser um fator fundamental. Alguns estudos sugerem que a criança desenvolve um estilo de relacionamento com o cuidador, que interfere no seu ajustamento à situação. Características do cuidador, especialmente empatia quanto às necessidades e sentimentos da criança e interesse pelas suas atividades, geram mais envolvirnento, propiciam brincadeiras mais complexas e mais atividades conjuntas entre as crianças (THYSSEN, 1995). A estabilidade do adulto também é importante: crianças sob alguma tensão tendem a iniciar mais comportamentos de apego a pagens estáveis e estas tendem a ser mais competentes para acalmar as crianças (BARNAS \& CUMMINGS, 1994). Além disso, características da própria creche também devem ser consideradas, como por exemplo, a proporção de crianças por educador: quanto menor esta proporção, maiores os níveis de conversa, brincadeira, toques e risadas (HOWES \& RUBENSTEIN, 1985).

Em síntese, a experiência da criança na creche é afetada por sua história prévia e pelas suas próprias variáveis individuais, dependendo também das inúmeras possíveis características da creche e do educador. Por sua vez, essa experiência extra-familiar afeta o desenvolvimento subseqüente, na medida em que passa a fazer parte essencial da vida sócio-afetiva da criança.

A análise detalhada das interações das crianças entre si e com os adultos na creche pode ser reveladora do tipo de ajustamento que cada indivíduo apresenta à situação. A presente pesquisa visou estudar os tipos de interações apresentados por crianças na faixa etária dos 4 anos, em uma creche pública estadual, com o objetivo de verificar a existência de possíveis padrões de interações, e portanto estabelecer correlações entre os vários aspectos do comportamento interacional das crianças. Atenção especial foi dada aos comportamentos afiliativos e agonísticos e às estratégias de resolução de conflitos, estudando-se relações entre interações positivas e negativas.

Muitos estudos de crianças nessa faixa etária apontam que os meninos apresentam mais comportamentos agressivos (ROOPARINE, 1984) e as meninas mais comportamentos afiliativos (WHALEY \& RUBENSTEIN, 1994). Em um estudo brasileiro sobre agrupamento de crianças em uma creche pública, AFFONSO (1986) demonstrou que as crianças se agrupavam por idade, na faixa entre os 2 e 4 anos, e que as mais velhas se agrupavam por sexo, tendo sido também encontrado efeito do tempo de creche. Fatores como sexo e tempo de experiência na creche serão considerados no presente trabalho.

É preciso notar que na situação de creche a criança fica em contato com muitas outras crianças, na maior parte do tempo. O conhecimento da dinâmica dessas relações e do significado desses padrões de comportamento pode contribuir para uma melhora do atendimento na creche. A identificação de indicadores comportamentais de dificuldades pode facilitar a constatação de problemas e a intervenção dos educadores.

\section{MÉTODO}

\section{Sujeitos}

Foram observadas 22 crianças, 14 meninas e 8 meninos, com idades entre 3 anos e 5 meses e 4 anos e 3 meses na época do início da observação. Dez crianças frequentavam a creche há 2 
anos, nove há três anos e três há quatro anos; todas frequentavam a creche em período integral. Conviviam, no mesmo ambiente, com crianças que frequentavam a creche por apenas um período e com crianças do jardim, alguns meses mais velhas.

O maternal era dividido em duas turmas, cada uma com uma pagem responsável, do mesmo modo que o jardim, o que totalizava 4 pagens em contato diário com os sujeitos; havia também uma outra pagem que cuidava das crianças de vez em quando ou se alguma das pagens permanentes faltasse.

\section{Procedimento}

As observações foram realizadas em situações de recreação livre, no período da tarde, durante o segundo semestre de 1997 . Nesse contexto, as crianças tinham a oportunidade de escolher entre brincar no pátio, que possuía trepa-trepas, tanques de areia, um escorregador e outros brinquedos (caixotes, baldes, bonecas); ou participar de atividades organizadas, às vezes com supervisão das pagens, como colagens, pinturas, massinhas, brinquedos de montar.

Foram realizadas gravações em vídeo, de modo a permitir o registro dos comportamentos pelo método do sujeito focal (ALTMAN, 1974). $\mathrm{O}$ foco da filmagem foi mantido em um sujeito de cada vez, por 10 minutos seguidos, sendo gravado também o contexto adjacente, para possibilitar a observação de todas as atividades ou interações nas quais o sujeito se envolvesse. Cada criança foi filmada em 6 ocasiões, distribuídas uniformemente, ao longo de 2 meses.

Para a contagem das frequências, as sessões foram divididas em períodos de 30 segundos. Cada período foi classificado quanto ao estado geral da criança, ou seja, tipo de envolvimento predominante dentre as 5 categorias abaixo definidas. Foram também registradas as frequências de ocorrência das demais categorias.

Através da análise dos registros, foram definidas as seguintes categorias:

Estados gerais: avaliados em função de qual dos seguintes padrões predominou, em cada período de 30 segundos do registro.

1. Interação Positiva: Contato, não necessariamente verbal, entre as crianças, envolvendo algum tipo de troca entre as duas: brincadeira, conversa, olhar correspondido.

2. Interação Negativa: Qualquer contato não amistoso, brigas, disputas.

3. Observação: Olhar com interesse para a atividade de outras crianças, para as educadoras ou para qualquer outra coisa.
4. Atividade Individual: Estar entretida em qualquer comportamento que envolva apenas a própria criança (com exceção do comportamento de observação); por exemplo: brincar sozinha, explorar algo como uma planta, fazer um desenho.

5. Sozinha Apática: Criança parece estar "isolada do que está acontecendo à sua volta”, com o olhar perdido.

\section{Padrões de comportamentos relacionados à agressão:}

1. Agressão Espontânea: Quando uma criança tem um comportamento agressivo (empurrar, bater, xingar), sem que existisse um antecedente imediato observável no registro, que pudesse ser considerado como desencadeador do padrão agressivo.

2. Disputa: Competição, rixa quando em situação de conflito; disputa de objeto, brinquedo.

3. Defender-se sem agredir o outro: Resolver uma situação conflituosa defendendo os próprios interesses, mas sem agredir o outro, por exemplo por meio de conversa.

4. Revide: Reagir a uma agressão com uma agressão física.

5. Não Reação: Não reagir diante de uma agressão ou conflito, ou simplesmente ceder ao que a outra criança quer.

\section{Padrões relacionados ao educador:}

1. Afeto: Qualquer comportamento pelo qual a pagem manifesto carinho pela criança.

2. Brincadeira de pai em com a criança: Comportamento de brincadeira ou conversa da pagem para com a criança.

Além dessas categorias, foi definida uma variável chamada de número de amigos: número de parceiros regulares com os quais o sujeito interagia; definidos quando existiam no mínimo um total de 10 interações positivas com a outra criança (incluindo as observações do sujeito focal e as da outra criança) ou 5 interações positivas quando a outra criança não era um sujeito focal.

Todas as análises foram feitas sobre os totais de frequência acumulados das 6 sessões para cada sujeito, tendo sido aplicados: 1 ) testes de correlações de Pearson e 2) testes t de comparação de médias para amostras independentes em função de sexo, tempo de creche e de algumas classificações comportamentais: as crianças foram subdivididas em três grupos em função do nível 
de interações positivas, de modo que, uma vez atribuídas as frequências, o terço inferior da distribuição foi designado como grupo de nível mínimo, o intermediário como médio e o superior como máximo. Os testes foram aplicados com a utilização do pacote estatístico "SPSS" (Statisti cal Package for Social Sciences).

\section{RESULTADOS E DISCUSSÃO}

De todas as categorias comparadas em função de sexo, foi constatado efeito tendendo a significativo em apenas uma: os meninos apresentaram mais revide do que as meninas ( $\mathrm{Xo}=1,38$; $\mathrm{Xa}=0,35 ; \mathrm{t}=2,07 ; \mathrm{p}=0,068)$. Este resultado, embora compatível, não chega a confirmar totalmente o dado proeminente da literatura sobre predominância de comportamentos afiliativos nas meninas e agressivos nos meninos, o que sugere cuidado na generalização desses achados e complexidade no efeito da variável sexo.

Tempo de creche também influenciou apenas a categoria brincadeira da pagem com a criança: as crianças com 4 anos de creche $(X=8,33)$ brincaram com a pagem mais do que as com 3 anos de creche ( $X=3,11 ; t=-2,97 ; p=0,014)$, e estas não se distinguiram das de 2 anos de creche $(X=4)$. Quanto à familiaridade, nenhuma de nossas crianças era realmente novata, sendo 2 anos o tempo mínimo de experiência naquela creche. No presente caso, esta variável também corresponde a diferentes idades de entrada da criança, Fato que também tem sido considerado relevante. Não se pode saber qual das 2 variáveis, o tempo de creche ou a idade de ingresso, afetou o padrão de brincadeira da pagem com a criança.

A análise dos padrões interacionais dos sujeitos, feita através do teste de correlações entre as categorias, revelou resultados interessantes.

Figura 1: Relação entre alguns comportamentos em cada criança

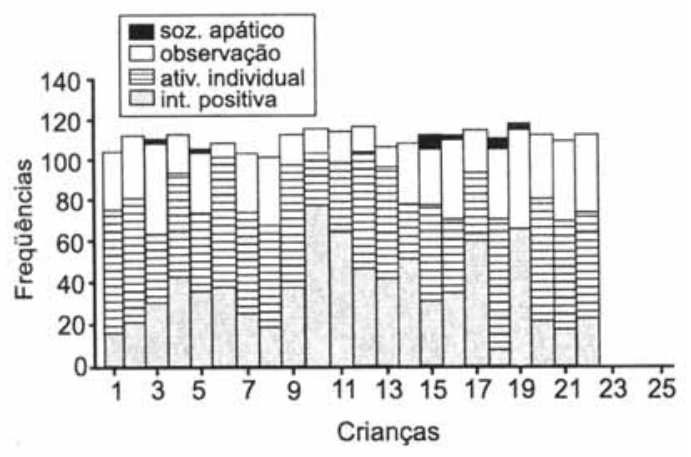

Vários comportamentos correlacionam-se com o nível de interações positivas exibidos pelas crianças. Histogramas representando as frequências destes comportamentos estão na Figura 1.

Há correlação positiva entre interações positivas e número de amigos $(\mathrm{R}=+0,67$; $p=0,001)$. As crianças que mais interagem positivamente são as que têm maior número de amigos. Essa relação não é óbvia porque uma criança poderia ter apenas um amigo com quem brincasse a maior parte do tempo e ainda assim exibir frequência alta de períodos de 30 segundos com interação positiva, nesse caso hipotético haveria correlação negativa entre as duas categorias.

Há correlação negativa entre interacção positiva e observação $(\mathrm{R}=0,72 ; \mathrm{p}=0)$. Essa relação negativa mostra que observar outra(s) criança(s) não é uma etapa anterior à interação pois, se esse fosse o caso, uma criança que tivesse altos índices em um desses comportamentos também o teria no outro. A correlação negativa entre observação e número de amigos $(\mathrm{R}=-0,50$; $\mathrm{p}=0,017$ ), parece confirmar ambas as correlações anteriormente expostas. Interação positiva e atividade individual também mostraram relações inversas $(R=-0,69 ; p=0)$, assim como a relação entre atividade individual e número de amigos $(\mathrm{R}=-0,44 ; \mathrm{p}=0,041)$. O comportamento sozinho apático exibiu correlação negativa com interação positiva $(R=-0,36$; $p=0,079)$ e com amigos $(R=-0,40 ; p=0,068)$ e correlação positiva com observação ( $\mathrm{R}=+0,40$; $\mathrm{p}=0,063)$.

$\mathrm{O}$ fato de existir correlação positiva entre observação e apatia e de ambos padrões estarem inversamente correlacionados com interação positiva, sugere que estes sejam indicadores de dificuldades na interação. Atividade individual, embora se correlacione negativamente com interação positiva, não apresenta correlação com observação e com apatia; sendo assim, merece ser considerada à parte. A figura 2 ilustra as médias desses padrões nos subgrupos mínimo, médio e máximo de interação positiva, podendo ser claramente observada a queda das frequências destas categorias em função do aumento do nível de interações positivas.

É interessante notar que alguns comportamentos relativos à agressão manifestaram-se mais frequentemente em crianças com níveis mais altos de interações positivas. Conforme pode ser visto na figura 3, as crianças do grupo de interação positiva mínima, destacaram-se por apresentar os níveis mais baixos de disputa $(X=0,42)$ diferindo significativamente do grupo médio $(\mathrm{X}=1,5 ; \mathrm{t}=-3,87 ; \mathrm{p}=0,002)$, o mesmo tendo acontecido em relação à agressão espontânea 
Figura 2: Grupos de Int. Positiva e Médias de alguns Comportamentos

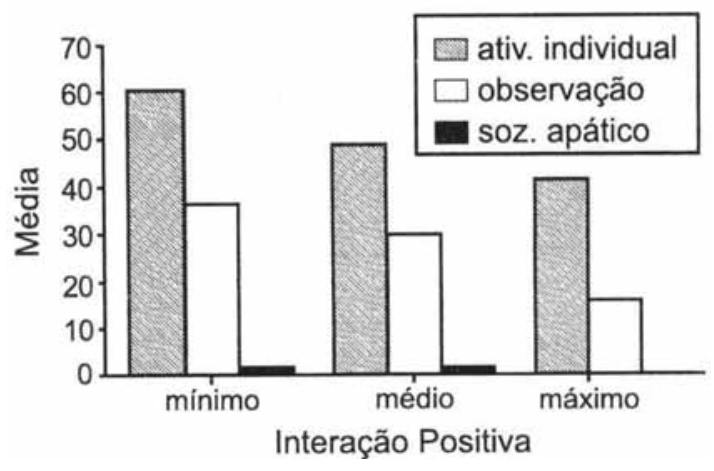

( $\mathrm{X}$ do nível mínimo $=0,29 ; \mathrm{M}$ do nível médio $=1,25 ; \mathrm{t}=-1,8 ; \mathrm{p}=0,1)$ e ao defender-se sem agredir o outro (X do nível mínimo $=0,42$; $\mathrm{X}$ do nível médio = 1,38; $\mathrm{t}=-2,03 ; \mathrm{p}=0,07)$. As crianças dos grupo mínimo e médio de interação positiva foram as que mais "não-reagiram" à agressão e tenderam a diferir significativamente do grupo máximo de interação positiva $(\mathrm{X}$ do nivel mínimo $=$ 1,85; $\mathrm{X}$ do nível médio = 2,25; $\mathrm{X}$ do nível máximo $=0,71$ sendo $t=1,96$ e 2,$14 ; p=0,075$ e 0,06 comparando-se os níveis mínimo e máximo, e médio e máximo respectivamente).

Figura 3: Grupos de Int. Positiva e Médias de Alguns Comportamentos

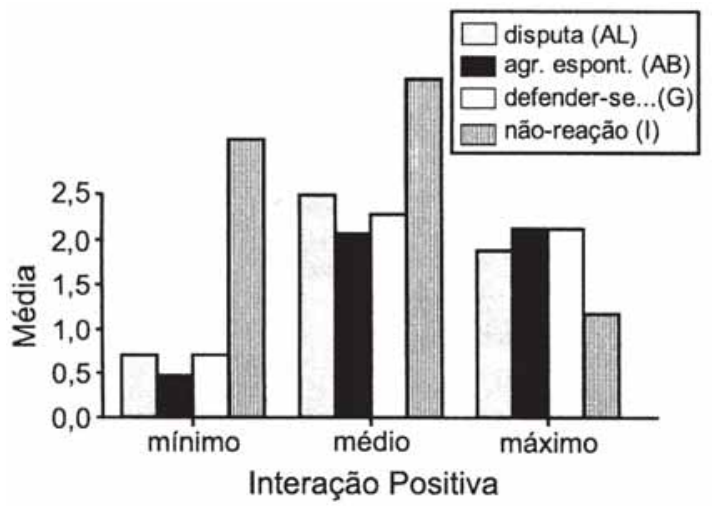

Embora não tenha sido encontrada correlação significativa entre interações negativas e positivas, estes resultados mostram que as crianças com baixos índices de interações positivas também tendem a apresentar baixos índices de alguns padrões agressivos. Isto parece decorrer mais de dificuldades gerais de relacionamento do que de afastamento do convívio social pois também é mais alto o índice em "não reagir à agressão", portanto, mesmo quando estas crianças estão claramente expostas a trocas sociais conflituosas, esses comportamentos não ocorrem, ou ocorrem com pouca frequência. Ainda que em níveis exagerados os padrões agressivos possam ser considerados desajustados, parece que a presença desses padrões, no caso, pode estar associada a melhor ajustamento.

Por sua vez, nessa amostra, baixos níveis de envolvimento em interações positivas não parecem estar relacionados a desinteresse social, pois estas crianças também tendem a apresentar muita observação das demais.

A busca de correlações entre os comportamentos gerais da criança e aqueles relacionados ao educador, nesta situação de recreação livre, revelou diferença significativa apenas quanto à correlação positiva entre "observar a pagem" e "afeto da pagem para com a criança” $(\mathrm{R}=0,38$; $\mathrm{p}=0,084)$. Esse resultado pode ser devido à uma maior proximidade física nessas crianças em relação as pagens, ou então, de algum modo pode ser que o adulto seja sensível ao interesse dessas crianças, o mesrno não ocorrendo com os seus pares, que não parecem ser atraídos pela respectiva observação. Deve-se ter em mente que esse observar a pagem correlacionou-se apenas com o afeto, mas não com outros comportamentos em relação à pagem.

\section{CONCLUSÃO}

Ao que tudo indica, nessa faixa etária, indices baixos de interação positiva aparecem associados a índices altos de observação, apatia e atividade individual, e a um menor número de amigos; estes constituindo-se em indicadores de dificuldades de interação e potencial dificuldade de ajustamento à creche.

A ocorrência de comportamentos relacionados à agressão (disputa, agressão espontânea, defender-se sem agredir o outro), em certa medida, pareceu nesse trabalho estar relacionada ao ajuste na creche. Ao contrário, a ausência ou escassez desses comportamentos aliada ao alto indice de nãoreação parece estar relacionada a dificuldades em interagir e talvez ao ajuste à creche.

Além disso, mostrou-se que observar a pagem está relacionado com o afeto recebido desta e algumas hipóteses foram levantadas para discutir essa relação.

Os resultados desse estudo levaram a uma questão sobre como seria o comportamento das crianças em situações mais dirigidas pela pagem; isso ajudaria a entender melhor a dinâmica das interações entre as crianças, e como se comportam nesse contexto criança que no caso da recreação livre pareceram ter dificuldades na interação com outras. 
De qualquer modo, a constatação desses padrões interacionais diversos aponta a necessidade de consideração desses fatos pelos edu- cadores para que possam ser criadas condições favoráveis ao desenvolvimento das crianças nas creches.

\begin{abstract}
Western parenting style increasingly includes day-care institutions. like kindergartens and daycare cent es. Based on developmental ethological studies, nowadays the social and aftective daily contexts are believed to be essential for the determination of human development, which explains the importance of investigating children behavior in these daily situations. The present paper examined interactional patterns displayed by four-year-old children in a public day-care centre, and investigatcd $\operatorname{co}^{\sim} \wedge \mathrm{Te} \wedge$ lations among the children's behaviors in order to evaluate social and affective styles of adjustrnent to the day-care institution. We observed 22 subjects in recreation time, videotaping sixten-minute sessions for each child as focal subject during two months. Results revealed significant negative correlations between: "positive interactions " and "observing others ", weakening a supposition of lack of interest to explain the low level of positive interaction; and between "positive interactions “ and apathy “, suggesting difficulties in the adjustment to the situation. On the other hand, children with a low level of "positive interac tion ' also sigruficantly displayed a low level of "aggressive patterns “. even when disturbed they reacted less than the other ones, indicating an interactional limitation instead of absence of social contact. In the present study, the results were not affected by the amount of previous experience in the day-care centre, maybe because there were no children with less than two years of experience, nor by sex, possibly due to the complexity of the interaction of this variable with the others.
\end{abstract}

Key-words: day-care centre; developmental context; interaction; children adjustment.

\section{REFERÊNCIAS BIBLIOGRÁFICAS}

AFFONSO, M. Redes sociais de um grupo de préescolares em creche institucionalizada: 1. Organização social. Psicologia,12(1): 5370, 1986.

ALTMAN, J. Observacional study of behavior: Sampling methods. Behavior, 49(314): 227267, 1974.

BARNAS, M.; CUMMINGS, E. Caregiver stability and toddler's attachment-related behavior towards caregivers in day-care. Infant Behavior Development, 17(2):141-148, 1994.

BOOTH, C.; KRASNOR, L.; RUBIN, K. Relating preschoolers' social competence and their mothers' parenting behaviors to early attachment security and high risk status. Journal of Social and Personal Relantionships, 8(3): 363-382, 1991.

BOWLBY, J. Apego. São Paulo, Martins Fontes Ed., 1984.

COHN, D. Child mother attachment of six year olds and social competence at school. Child Development, 61(1): 152-162, 1990.

HOWES, C.; RUBENSTE1N, J. Determinants oftoddlers' experience in day-care: age of entry and quality of setting. Child Care Quarterly, 14(2): 140-151, 1985.
KERNS, K.; BARTH, J. Attachment and play: Convergence across components of parentchild relationships and their relations to peer competence. Journal of Social and Personal Relationships, 12(2): 243-260, 1995.

MATAS, L.; AREND, R.; SROUFE, L. Continuity of adaptation in the second year: The relationship between quality of attachment and later competence. Child Development, 49(3): 547-556, 1978.

RENKEN, B.; EGELAND, B.; MARVINNEY, D.; MANGELSDORF, S.; STROUFE, L. Early childhood antecedents of agression and passive withdrawal in early elementary school. Journal of Personality, 57(2): 257-282, 1989.

ROOPARINE, J. Sex-typed socialization in mixed-age preschool classroom. Child Development, 55(3): 1078-1084, 1984.

THYSSEN, S. Care for children in day care centers. Child and Youth Care Forum, 24(2): 91-106, 1995.

WHALEY, K. L.; RUBENSTEIN, T. S. How toddlers "co" friendship: A descriptive analysis of naturally ocurring friendships in a group child case setting. Journal of Social and Personal Relationships, 11(3): 383-400, 1994. 